\title{
Common vestibular disorders in children in Fayoum governorate: a cross-sectional study
}

Hossam Abd Elghaffar', Sherif Guindi², Mahmoud Mohamed Magdy², Mona Alakkad² and Reham Rafei El Shafei ${ }^{2^{*}}$ (1)

\begin{abstract}
Background: Vertigo in paediatric age is not a rare condition. However, a lack of awareness about causes of dizziness in children besides the absence of standard diagnostic workup often leads to unnecessary procedures in managing these cases. The purpose of this cross-sectional study was to determine the prevalence and frequency distribution of balance abnormalities in dizzy children. Additionally, the questionnaire's utility as a screening tool and its predictive accuracy are discussed. Thus, an organized strategy can be allocated to assist the diagnostic process, which is likely to eliminate unnecessary and costly evaluations.

Results: This study comprised 848 children who presented with any of the dizzy symptoms referred to the AudioVestibular Unit, Fayoum University Hospital. The suggested diagnosis was $23.9 \%$ of the cases suffered from otitis media either alone or combined with other diseases, while 17.6\% had benign paroxysmal vertigo of childhood. Migraine patients represented $11.2 \%$ of the cases. Only $5.5 \%$ of subjects had benign paroxysmal positional vertigo. $78.3 \%$ of cases with possible diagnosis showed agreement in diagnosis by both paediatric dizziness questionnaire and the final diagnosis reached by a full assessment of the patients.
\end{abstract}

Conclusion: Diagnosing dizziness in children is challenging; nevertheless, a structured history taken in conjunction with a vestibular examination is crucial for establishing an accurate diagnosis. The questionnaire appears to be an even-handed tool for diagnosing dizzy children.

Keywords: Vertigo, Dizziness, Questionnaires, Audio-vestibular assessment, Dizzy child

\section{Background}

Dizziness is a common complaint that refers to various symptoms such as unsteadiness, imbalance, clumsiness, lightheadedness, and vertigo. Nonetheless, vertigo is a medical word that refers to a subjective or objective perception of movement that is frequently connected with vestibular system disorders [1].

Vertigo in paediatric age is not a rare condition, with about a $10 \%$ prevalence in most clinical trials; yet, young children are frequently unable to explain these different perceptions [2]. Vestibular disorders are recognized as

\footnotetext{
*Correspondence: rrs00@fayoum.edu.eg; rehamrafei@yahoo.com

2 ENT Department, Faculty of Medicine, Fayoum University,

Fayoum 63514, Egypt

Full list of author information is available at the end of the article
}

the most common causes of dizziness in children, with a prevalence of $0.7-15 \%$. However, the actual incidence of vertigo in the paediatric group remains underestimated due to the lack of exact clinical classification and solid diagnostic workup [3-6].

In addition to that, these disorders may often remain undiagnosed in children [7] as medical professionals may attribute symptoms to behavioural confusion or 'clumsiness' [8]; significantly, children may demonstrate behaviours such as clinging to the parent when experiencing dizziness or vertigo [9]. Above and beyond, vertigo in children constantly creates a profound sense of anxiety both in parents and physicians, leading to the excessive number of prescriptions for functional testing and imaging examinations [10]. 
Accordingly, lack of awareness about causes of dizziness in children besides the absence of standard diagnostic workup often leads to unnecessary procedures in managing these cases [4]. The purpose of this crosssectional study was to determine the prevalence and frequency distribution of balance abnormalities in dizzy children. Additionally, the questionnaire's utility as a screening tool and its predictive accuracy are discussed. Thus, an organized strategy can be allocated to assist the diagnostic process, which is likely to eliminate unnecessary and costly evaluations.

\section{Methods}

\section{Subjects}

\section{The study design and settings}

The purpose of this cross-sectional study was to determine the prevalence and frequency distribution of balance abnormalities in dizzy children. Additionally, the questionnaire's utility as a screening tool and its predictive accuracy are discussed. Thus, an organized strategy can be applied to assist the diagnostic process, which is likely to eliminate unnecessary and costly evaluations.

The study included 1007 children suffering from dizziness or vertigo. The study group underwent basic audiological and vestibular test batteries and filled in the questionnaire with their parents before testing. This study was conducted for four years, from August 2016 till February 2020, at the Audio-Vestibular Unit, University hospital.

Inclusion criteria:

1- Dizzy child presented by any following symptoms: unsteadiness, imbalance, clumsiness, light-headedness, or vertigo.

2- Age group between 6 and 18 years (females or males).

\section{Exclusion criteria:}

1- Any child who refused to complete testing (either child or guardian).

2- Any child with a known neurological disorder.

All subjects underwent

1- A thorough medical history taking: It includes a complete description of the dizziness complaint regarding frequency, duration, progression, and character. Also, precipitating and relieving factors were included. Any accompanying symptoms and medication received were documented.
2- Paediatric Dizziness Questionnaire [11]. (Shabana, M.I., Dabbous, A.O., Hosni, N.A. et al. (2012): Can scoring of symptoms in dizzy children aid the categorization of causes of dizziness for accurate referral? Egypt J Otolaryngol 28, 214-233 https://doi.org/10. 7123/01.EJO.0000418071.01257.ba) (Additional file 1).

Children and guardians filled it in. The questionnaire was developed in the light of a good knowledge of causes of childhood dizziness and with the help of previously validated questionnaires [11]: It included the following: (a) description of dizziness, (b) relevant medical history, (c) effect of dizziness on school, educational, and daily activities, (d) developmental and educational history, (e) relevant past medical history (and system review), and (f) family history. The questions were grouped into seven diagnostic categories reflecting the different systems involved in balance control, overlapping some category questions. There were 24 questions in each vestibular and neurological category; eight questions in the general classification; four questions in each of the cervical and ocular categories; six questions in the cardiovascular (CVS) category; and three questions in the psychological category. Therefore, a common denominator of 24 was used for our scoring system, with a total of 24 points for each of the seven major categories. Accordingly, the vestibular category was assigned 1 point for a 'yes' answer in each of the 24 questions and was defined as associated or not with hearing loss. The neurological category was assigned 1 point for a 'yes' answer for every 24 questions. The general category was given 3 points for a 'yes' answer for each of the eight questions. CVS category was assigned points for a 'yes' answer in each of the eight questions. The cervical category was given 6 points for a 'yes' answer for each of the four questions. The ocular category was assigned 6 points for a 'yes' answer for each of the four questions. The psychological category was given 8 points for a 'yes' answer for each of the three questions. A percentage of impairment score was obtained, and the total scoring of each system was described. The conclusions of the questionnaire were built based on the present history results of each category score, aided by any relevant data provided in the past medical or family history and system review. Thus, a conclusion was reached from the questionnaire for each child, that is, on an individual basis, which led to a particular direction in diagnosis. The referral results to different clinics were used to assess the questionnaire's ability to assign dizziness into diagnostic categories.

Scoring Points assign to 'Yes' answers only.

Score of $100=$ normal, and as score decreases, the system affection increases. 
Questions with overlapping of categories assign points for each category.

\begin{tabular}{llll}
\hline Category & $\begin{array}{l}\text { Number of } \\
\text { items }\end{array}$ & $\begin{array}{l}\text { Each item } \\
\text { scores }\end{array}$ & Total Score \\
\hline Vestibular & 24 & 1 & 24 \\
Neurological & 24 & 1 & 24 \\
General & 8 & 3 & 24 \\
CVS & 6 & 4 & 24 \\
Ocular & 6 & 4 & 24 \\
Psychological & 3 & 8 & 24 \\
\hline
\end{tabular}

3- Basic audiological evaluation:

- Pure tone audiometry: air conduction thresholds were tested at frequencies between 250 and 8000 $\mathrm{Hz}$ at octave intervals. Bone conduction thresholds were tested at frequencies between 500 and $4000 \mathrm{~Hz}$, also at octave intervals with contralateral masking using narrow-band noise.

- Speech audiometry: Speech reception threshold (SRT) [12] and word discrimination scores (WDS) [13].

- Acoustic immittance testing: this included tympanometry and acoustic reflexes (ipsilateral and contralateral) using tone stimuli at 500, 1000, 2000, and $4000 \mathrm{~Hz}$. The frequency of the probe used is $226 \mathrm{~Hz}$.

4- Bedside examination:

a. Observing the child's gait and stance.

b. Romberg test: A Romberg test assesses the integrity of peripheral proprioception, cerebellar and vestibular functions [14].

c. Fukuda test: With unilateral vestibular lesions, this manoeuvre will elicit veering toward the affected side by more than $45^{\circ}$. With bilateral vestibular dysfunction, a significant forward displacement of more than 20 inches may be seen [15].

d. Dix-Hallpike positioning test: Turn the patient's head 45 degrees to one side\& lay the patient supine with their head over the end of the examination bed, supporting their head with a hand on each side of the head. The result is positive if the patient develops symptoms (vertigo) and nystagmus, then repeat on the opposite side [14].

e. The head thrust test is a simple clinical manoeuvre that detects total unilateral or bilateral vestibular loss. The child fixes gaze on the nose of the examiner, who holds the child's head between their hands and then makes a very rapid (high-acceleration) $30^{\circ}$ rotational movement to the right or left ('head thrust'), asking the child to keep the gaze fixed on the examiner's nose. When the head is rapidly turned toward the side of absent vestibular function, one or more 'catch up' saccades is observed.

Videonystagmography (VNG) VNG was performed using two-channel monocular Micromedical Mobile Eyes, where the camera can image the eye via a specialized mirror. Calibration was mandatory for accurate nystagmus recording. VNG subtests were occulography tests (smooth pursuit, saccade, and optokinetic), spontaneous nystagmus, gaze, positional, positioning, and caloric tests. The caloric test was in the following sequence: right cool, left cool, right warm, and left warm. The irrigation lasted for 40 seconds using an air caloric irrigator.

Temperatures were $25^{\circ} \mathrm{C}$ for cold air and $49^{\circ} \mathrm{C}$ for warm air; the airflow rate was maintained at 8 liters per minute. Subjects were asked to perform a mental task to maximize the response during the recording. A software algorithm was used automatically to calculate unilateral weakness, directional preponderance, and total eye velocity using standard formulae. Threshold values for caloric testing according to Bárány Society Consensus document [16]: unilateral weakness greater than (20 to $25 \%$ ) is significant. Average caloric responses of a total caloric response $<12 \%$ are diagnostic of bilateral deficiency.

Equipment:

a. Audiometry: Interacoustics AC40 calibrated according to the ISO standards.

b. Immitancemeter: Interacoustics AT235 calibrated according to the ISO standards.

c. Videonystagmography: computerized 2-channels VNG Micromedical.

The vertigo diagnoses were based on standard published criteria. For benign paroxysmal vertigo of childhood, we used The International Classification of Headache Disorders, 2018 [17].

\section{Diagnostic criteria:}

A. At least five attacks fulfilling criteria B and C 
B. Vertigo occurring without warning, maximal at onset and resolving spontaneously after minutes to hours without loss of consciousness

C. At least one of the following five associated symptoms or signs:

1. Nystagmus.

2. Ataxia.

3. Vomiting.

4. Pallor.

5. Fearfulness.

D. Normal neurological examination and audiometric and vestibular functions between attacks

E. Not attributed to another disorder.

Diagnostic criteria used for vestibular migraine according to The International Classification of Headache Disorders 3rd edition 2018 [17].

A. At least five episodes fulfilling criteria C and D

B. A current or history of migraine without aura or migraine with aura.

C. Vestibular symptoms of moderate or severe intensity, lasting between 5 minutes and 72 hours

D. At least half of episodes are associated with at least one of the following three migrainous features:

1. Headache with at least two of the following four characteristics:
a. Unilateral location.
b. Pulsating quality.
c. Moderate or severe intensity.
d. Aggravation by routine physical activity.

2. Photophobia and phonophobia.

3. Visual aura.

E. Not better accounted for by another ICHD-3 diagnosis or another vestibular disorder.

We required typical case medical history and a positive finding in the Dix Hallpike maneuver to diagnose benign paroxysmal positional vertigo. Vestibular neuronitis was diagnosed based on a sudden onset of severe rotatory vertigo, spontaneous horizontorotatory nystagmus, and a lack of neurologic signs that could indicate central nervous system involvement.

For Ménière's disease (MD), we used the American Academy of Otolaryngology-Head and Neck Foundation criteria, 2014 [18].

\section{Statistical analysis}

Data were collected and coded to facilitate data manipulation and double entered into Microsoft Access. Data analysis was performed using Statistical Package of Social Science (SPSS) software version 18 in windows.

Simple descriptive analysis in numbers and percentages for qualitative data, arithmetic means as central tendency measurement, standard deviations as a measure of dispersion for quantitative parametric data.

Quantitative data included in the study was first tested for normality by the One-Sample Kolmogorov-Smirnov test in each study group, then inferential statistic tests were selected.

\section{For quantitative parametric data}

In-depended Student's $t$-test used to compare measures of two independent groups of quantitative data

\section{For qualitative data}

Chi-square test to compare two of more than two qualitative groups. The $p$-value $\leq 0.05$ was considered the cutoff value for significance.

\section{Results}

A cross-sectional study design was implemented to assess the prevalence and frequency distribution of balance disorders in dizzy children referred to the Audio-Vestibular Unit, Fayoum University hospital and the role of questionnaire to serve as a screening tool and its accuracy to predict the diagnosis during four years from August 2016 till February 2020.

This study comprised 1007 children who presented with any of the following dizzy symptoms; unsteadiness, imbalance, clumsiness, light-headedness, or vertigo. However, 159 children refused to continue vestibular testing while conducting this study. They mainly refused to complete caloric testing and ocular motor assessment.

The study group consisted of 848 children, ranging from 6 to 18 years with a mean age of $10.86 \pm 3.7$ years, suffering from dizziness. They included 407 males and 441 females. Regarding sex distribution, $52 \%$ of the study group were females versus $48 \%$ males, with $30.5 \%$ showing positive family history, as shown in Table 1 excluding children who refused to continue the tests.

The description of different Peadiatric Dizziness Questionnaire items scores; first, the vestibular category the mean was $(8.3 \pm 3)$ ranged between 2 and 19, the neurological part the mean was $(5.6 \pm 3.7)$ ranged between 1 and 15, as regards general items the mean was (7.3 \pm 4.9) ranged between 0 and 24, for CVS the mean was ( 4.2 \pm 4.2 ) ranged between 0 and 16 , for the ocular category the mean, was $(3.2 \pm 4.6)$ ranged between. Finally, for the psychological part, the mean was $(3 \pm 5.5)$ ranged 
Table 1 Description of demographic characters among the study group

\begin{tabular}{lll}
\hline Variables & Number $(\boldsymbol{n}=\mathbf{8 4 8})$ & \\
\hline Age (years) & & \\
$\quad$ Mean /SD & 10.86 & 3.7 \\
Sex & & $48 \%$ \\
$\quad$ Male & 407 & $52 \%$ \\
$\quad$ Female & 441 & \\
Family history & & $69.5 \%$ \\
$\quad$ Negative & 589 & $30.5 \%$ \\
$\quad$ Positive & 259 & \\
\hline
\end{tabular}

Table 2 Description of differences of Pediatric Dizziness Questionnaire categories scores among the study group

\begin{tabular}{llll}
\hline Score items & Mean & SD & Range \\
\hline Vestibular & 8.3 & 3 & $2-19$ \\
Neurological & 5.6 & 3.7 & $1-15$ \\
General & 7.3 & 4.9 & $0-24$ \\
CVS & 4.2 & 4.2 & $0-16$ \\
Ocular & 3.2 & 4.6 & $0-24$ \\
Psychological & 3 & 5.5 & $0-24$ \\
Total score & 31.6 & 18.4 & $8-87$ \\
\hline
\end{tabular}

between 0 and 24, as regards total score, the mean was $(31.6 \pm 18.4)$ ranged between 8 and 87 as shown in Table 2.

The basic audiological evaluation comprised both PTA \& tympanometry. As for PTA results: $24.9 \%$ of the study group had conductive hearing loss, either unilateral $(8 \%)$ or bilateral (16.9\%), followed by $12.7 \%$ for sensorineural hearing loss, either unilateral $(4.8 \%)$ or bilateral $(7.9 \%)$ and $0.7 \%$ for mixed hearing loss Table 3. For the degree of hearing loss, most cases showed a mild degree of hearing loss, $64.4 \%$ on the right side versus $66.1 \%$ on the left side. Also, $18.1 \%$ of them had a moderate degree of hearing loss on the right side versus $16.2 \%$ on the left side, and $17.4 \%$ of them showed a severe degree of hearing loss on the right side versus $17.7 \%$ on the left side, as shown in Table 4 . Tympanometry could not be done in $1.5 \%$ of the study group on the right side versus $2.6 \%$ on the left side. $80.9 \%$ showed type A tympanogram on the right side, versus $77.4 \%$ on the left side, type B represented $14.6 \%$ of the study group on the right side versus $16.6 \%$ on the left side, for type $C$ tympanogram, it represented $2.9 \%$ on the right side and $3.4 \%$ on the left side as shown in Table 5.

Regarding VNG testing results: only $3.3 \%$ of cases (28 children) showed spontaneous nystagmus. Nineteen
Table 3 Types of hearing loss in the study group

\begin{tabular}{llll}
\hline & \multicolumn{2}{c}{ study } & Ears \\
\cline { 2 - 3 } & No. & $\%$ & \\
\hline Normal & 523 & $61.7 \%$ & 1155 \\
Unilateral CHL & 68 & $8 \%$ & 68 \\
Bilateral CHL & 143 & $16.9 \%$ & 286 \\
Unilateral SNHL & 41 & $4.8 \%$ & 41 \\
Bilateral SNHL & 67 & $7.9 \%$ & 134 \\
Bilateral mixed & 6 & $0.7 \%$ & 12 \\
Total & 848 & $100 \%$ & 1696 \\
\hline
\end{tabular}

Table 4 Type and degree of hearing loss in the study group

\begin{tabular}{|c|c|c|}
\hline \multirow[t]{2}{*}{ Variables $(n=848)$} & \multicolumn{2}{|c|}{ PTA results } \\
\hline & Number & $\%$ \\
\hline \multicolumn{3}{|l|}{ Right side hearing } \\
\hline Normal & 578 & $68.2 \%$ \\
\hline Conductive & 162 & $19.1 \%$ \\
\hline Sensorineural & 102 & $12 \%$ \\
\hline Mixed & 6 & $0.7 \%$ \\
\hline \multicolumn{3}{|l|}{ Left side hearing } \\
\hline Normal & 577 & $68 \%$ \\
\hline Conductive & 192 & $22.7 \%$ \\
\hline Sensorineural & 73 & $8.6 \%$ \\
\hline Mixed & 6 & $0.7 \%$ \\
\hline \multicolumn{3}{|c|}{ Degree of hearing loss on the right side $(n=270)$} \\
\hline Mild & 174 & $64.4 \%$ \\
\hline Moderate & 49 & $18.1 \%$ \\
\hline Severe & 47 & $17.4 \%$ \\
\hline \multicolumn{3}{|c|}{ Degree of hearing loss on the left side $(n=271)$} \\
\hline Mild & 179 & $66.1 \%$ \\
\hline Moderate & 44 & $16.2 \%$ \\
\hline Severe & 48 & $17.7 \%$ \\
\hline
\end{tabular}

were of peripheral origin versus 9 of them of central source. Horizontal-torsional spontaneous nystagmus in peripheral vestibular disorders is suppressed by fixation. Nystagmus, purely horizontal, vertical, or torsional, usually has a central cause [19].

During conducting the positional tests (head supine, head right \& head left), 83 cases only (9.8\%) had nystagmus, 34 cases of them, the positional nystagmus was the only positive finding, while the remaining cases, most of them were suffering from bilateral mild conductive hearing loss; either otitis media with effusions or chronic suppurative otitis media. $62.7 \%$ of patients showed right beating nystagmus on the head left versus $37.7 \%$ showed left beating nystagmus on head right. 
Table 5 Frequency of different tympanometry results among the study group

\begin{tabular}{lll}
\hline Variables $(\boldsymbol{n}=\mathbf{8 4 8})$ & \multicolumn{2}{l}{ Tympanometry results } \\
\cline { 3 - 3 } & Number & $\%$ \\
\hline Right side tympanometry & & \\
$\quad$ Not done & 13 & $1.5 \%$ \\
Type A & 686 & $80.9 \%$ \\
Type B & 124 & $14.6 \%$ \\
Type C & 25 & $2.9 \%$ \\
Left side tympanometry & & \\
Not done & 22 & $2.6 \%$ \\
Type A & 656 & $77.4 \%$ \\
Type B & 141 & $16.6 \%$ \\
Type C & 29 & $3.4 \%$ \\
\hline
\end{tabular}

Positive Dix-Hallpike test (head hanging to the right\& head hanging to the left) was recorded in only 47 cases (5.54\%). Torsional up-beating nystagmus on the right Dix-Hallpike in 25 cases versus torsional up-beating nystagmus on the left Dix-Hallpike in 22 cases.

Only $8.6 \%$ of cases showed abnormal saccades (slow velocity, inaccuracy), and $8.4 \%$ showed low gain smooth pursuit and asymmetry. Finally, $8.4 \%$ of cases had low gain OPK, as shown in Table 6.

According to Bárány Society Consensus document [16]:185 cases (21.8\%) had canal paresis on caloric testing: 108 patients showed right canal paresis while 77 patients showed left canal paresis as shown in Table 7 . Only 5 cases showed bilateral vestibular weakness; ototoxic drugs caused two of them. One case was associated with autoimmune disease, one case was associated with cerebellar degeneration and other neurological disorders, and the last one was idiopathic. Unilateral weakness is indicative of a peripheral vestibular lesion that involves the nerve or end-organ on

Table 6 Ocular-motor assessment among the study group

\begin{tabular}{lll}
\hline Variables & \multicolumn{2}{l}{ Ocular-motor assessment } \\
\cline { 3 - 3 } & Number & $\%$ \\
\hline Saccade & 775 & $91.4 \%$ \\
$\quad$ Normal & 73 & $8.6 \%$ \\
$\quad$ Slow velocity, inaccuracy & & \\
Smooth pursuit & 777 & $91.6 \%$ \\
$\quad$ Normal & 71 & $8.4 \%$ \\
Low gain and asymmetry & & \\
OPK & 777 & $91.6 \%$ \\
$\quad$ Normal & 71 & $8.4 \%$ \\
$\quad$ Low gain and asymmetry &
\end{tabular}

Table 7 Canal weakness among the study group

\begin{tabular}{lll}
\hline Variables $(\boldsymbol{n}=\mathbf{8 4 8})$ & \multicolumn{2}{l}{ Caloric test assessment } \\
\cline { 2 - 3 } & Number & $\%$ \\
\hline Normal & 658 & $77.6 \%$ \\
Right canal weakness & 108 & $12.7 \%$ \\
Left canal weakness & 77 & $9.1 \%$ \\
Bilateral vestibular weakness & 5 & $0.6 \%$ \\
\hline
\end{tabular}

the side of the weakness. In many clinics, a unilateral weakness greater than $25 \%$ is significant. Average caloric responses of six degrees per second or less are consistent with a bilateral weakness.

The suggested diagnosis reached was that 23.9\% (203 cases) of the cases suffered from otitis media-related dizziness either alone (94 cases) or combined with other diseases (109 cases). In comparison, 17.6\% (149 cases) of the cases had benign paroxysmal vertigo of childhood. Migraine patients represented $11.2 \%$ (95 cases) of the cases. Only $5.5 \%$ of cases (47 cases) had benign paroxysmal positional vertigo, versus $4.4 \%$ of cases (37 cases) had vestibular neuritis or labyrinthitis. Less common causes as cholesteatoma, ototoxicity, perilymph fistula, enlarged vestibular aqueduct, Meniere, and labyrinthine hydrops were collectively represented in less than $3 \%$ of cases. Neurological causes represented $6.6 \%$ of cases (56 cases) as follow: epilepsy (2.2\%), benign intracranial hypertension $(1.4 \%)$, brain tumours $(0.94 \%)$, meningitis $(0.82 \%)$, Arnold Chiari malformations (0.35\%), multiple sclerosis $(0.12 \%)$, stroke $(0.12 \%)$ and other neurological disorders $(0.59 \%)$ as shown in Table 8.

General causes as anaemia, DM, and other endocrinal disorders represented $13.9 \%$ of cases (118 cases) either alone (56 cases) or combined with other diseases (62 cases). Cardiovascular disorders were found in 3.1\% of cases (26 cases) either alone ( 14 cases) or combined with other conditions (12 cases), while ocular disorders were found in $1.8 \%$ (15 cases) of the cases. We could not reach a possible diagnosis in $20.5 \%$ of cases (174 cases), as shown in Table $8.20 .5 \%$ of patients had unexplained dizziness, versus $79.5 \%$ had causes for dizziness as follows: $41.5 \%$ had only vestibular causes, $6.6 \%$ of them had only neurological causes, and the same percentage for general causes, followed by $1.7 \%$ for cardiovascular causes, $12.9 \%$ showed combined vestibular and neurological causes, 7.3\% had connected vestibular and general causes, $1.5 \%$ for combined vestibular and ocular disorders, and finally $1.4 \%$ for the combination of vestibular and cardiovascular diseases with total percent of $23.1 \%$ for different combined causes as shown in Fig. 1.

According to the paediatric dizziness questionnaire, $36.2 \%$ of cases were diagnosed as vestibular dizziness, 
Table 8 Diagnosis among the study group

\begin{tabular}{lll}
\hline Variables & $\begin{array}{l}\text { Number }(\boldsymbol{n}= \\
\mathbf{8 4 8})\end{array}$ \\
\hline Otitis media-related dizziness & 203 & $23.9 \%$ \\
Benign paroxysmal vertigo of childhood & 149 & $17.6 \%$ \\
Migraine & 95 & $11.2 \%$ \\
Benign paroxysmal positional vertigo & 47 & $5.5 \%$ \\
Vestibular neuritis or labyrinthitis & 37 & $4.4 \%$ \\
cholesteatoma & 8 & $0.9 \%$ \\
ototoxicity & 5 & $0.6 \%$ \\
Perilymph fistula & 5 & $0.6 \%$ \\
Enlarged vestibular aqueduct & 4 & $0.5 \%$ \\
Meniere or labyrinthine hydrops & 3 & $0.35 \%$ \\
General & 118 & $13.9 \%$ \\
Cardiovascular & 26 & $3.1 \%$ \\
Ocular & 15 & $1.8 \%$ \\
Epilepsy & 19 & $2.2 \%$ \\
Benign intracranial hypertension & 12 & $1.4 \%$ \\
Brain tumours & 8 & $0.94 \%$ \\
Meningitis & 7 & $0.82 \%$ \\
Arnold Chiari malformations & 3 & $0.35 \%$ \\
Multiple sclerosis & 1 & $0.12 \%$ \\
Stroke & 1 & $0.12 \%$ \\
Other neurological disorders & 5 & $0.59 \%$ \\
Unexplained & 174 & $20.5 \%$ \\
\hline & &
\end{tabular}

$15.1 \%$ for general causes, $10.5 \%$ for psychological causes, $5.8 \%$ for neurological causes, and a lower percentage of $3.3 \%$ for ocular and $1.7 \%$ for cardiovascular causes. For combined causes, the results were as follow $13.7 \%$ for vestibular and neurological, followed by $7.4 \%$ for vestibular and general and lower percentages $1.4 \%, 1.7 \%, 0.9 \%, 0.8 \%$, $0.8 \%$, and $0.7 \%$ for combined ocular and vestibular, general and cardiovascular, general and ocular, neurological and general, neurological and psychological, cardiovascular and ocular respectively, with a total percentage of $27.5 \%$ (233 cases) for combined causes as shown in Fig. 2.

Figure 3 showed that $47.7 \%$ of cases with unexplained dizziness were categorized as psychic patients by paediatric dizziness questionnaire, which may suggest the need for a psychiatrist to assess unexplained dizzy children. Table 9 illustrates that there is a statistically significant difference with $p$-value $<0.05$ between explained and unexplained groups as regards hearing loss with a higher percentage of normal PTA on both sides among patients with unexplained dizziness and a higher percentage of conductive hearing loss on both sides among patients with possible diagnosis, most of them were due to otitis media.

Table 10 illustrates a statistically significant high mean score of different categories by paediatric dizziness questionnaire with $p$-value $<0.05$ among patients with unexplained dizziness.78.3\% of cases with possible diagnosis showed agreement in diagnosis by both paediatric dizziness questionnaire and the final diagnosis reached by a full assessment of the patients, as shown in Table 11, which emphasizes the importance of paediatric dizziness questionnaire in the evaluation of dizzy children (Fig. 4).

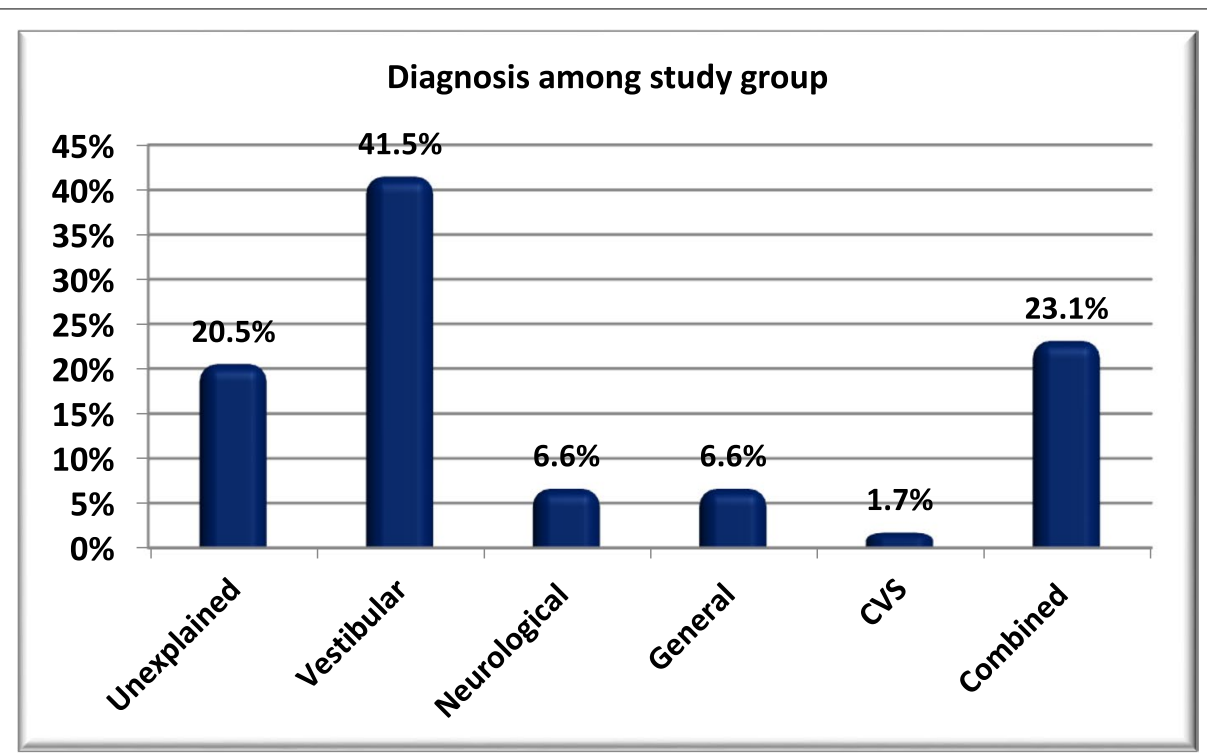

Fig. 1 Diagnosis among the study group by category 


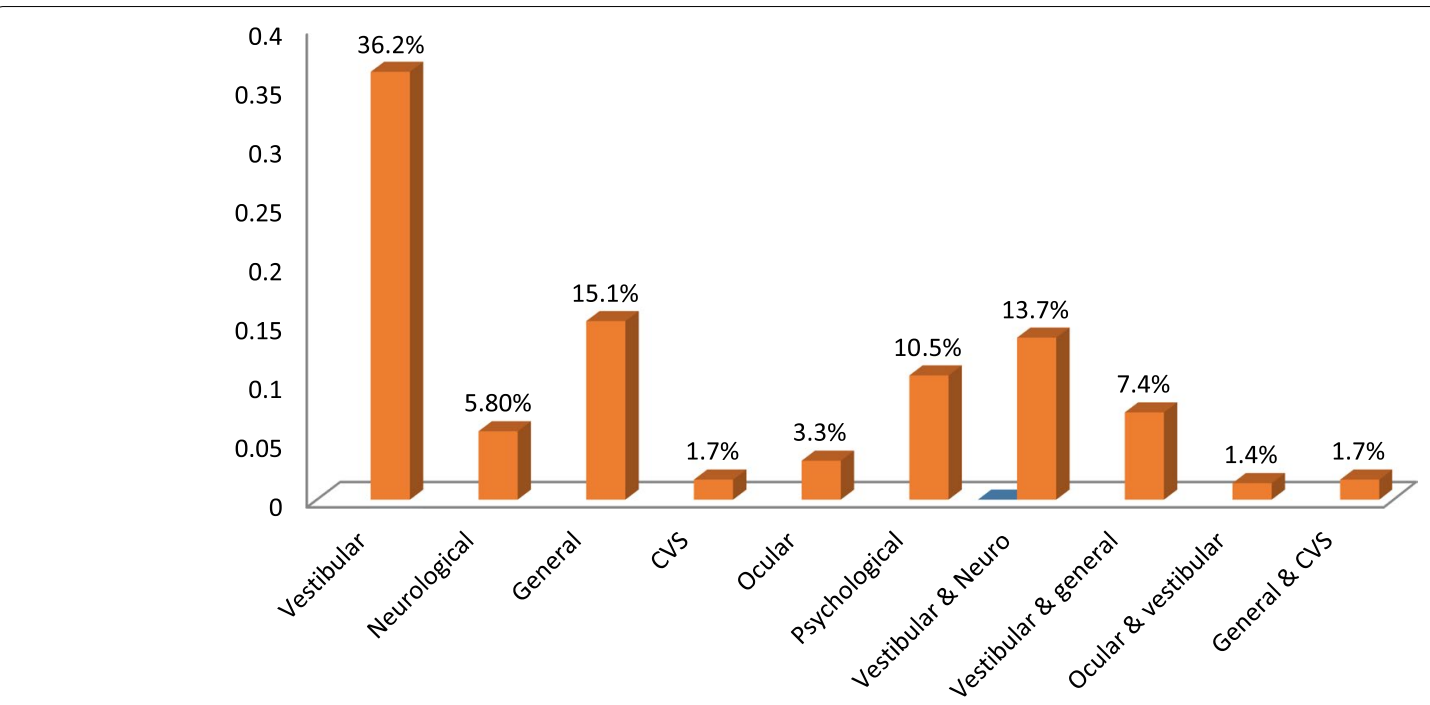

Fig. 2 Diagnosis by Pediatric Dizziness Questionnaire among the study group

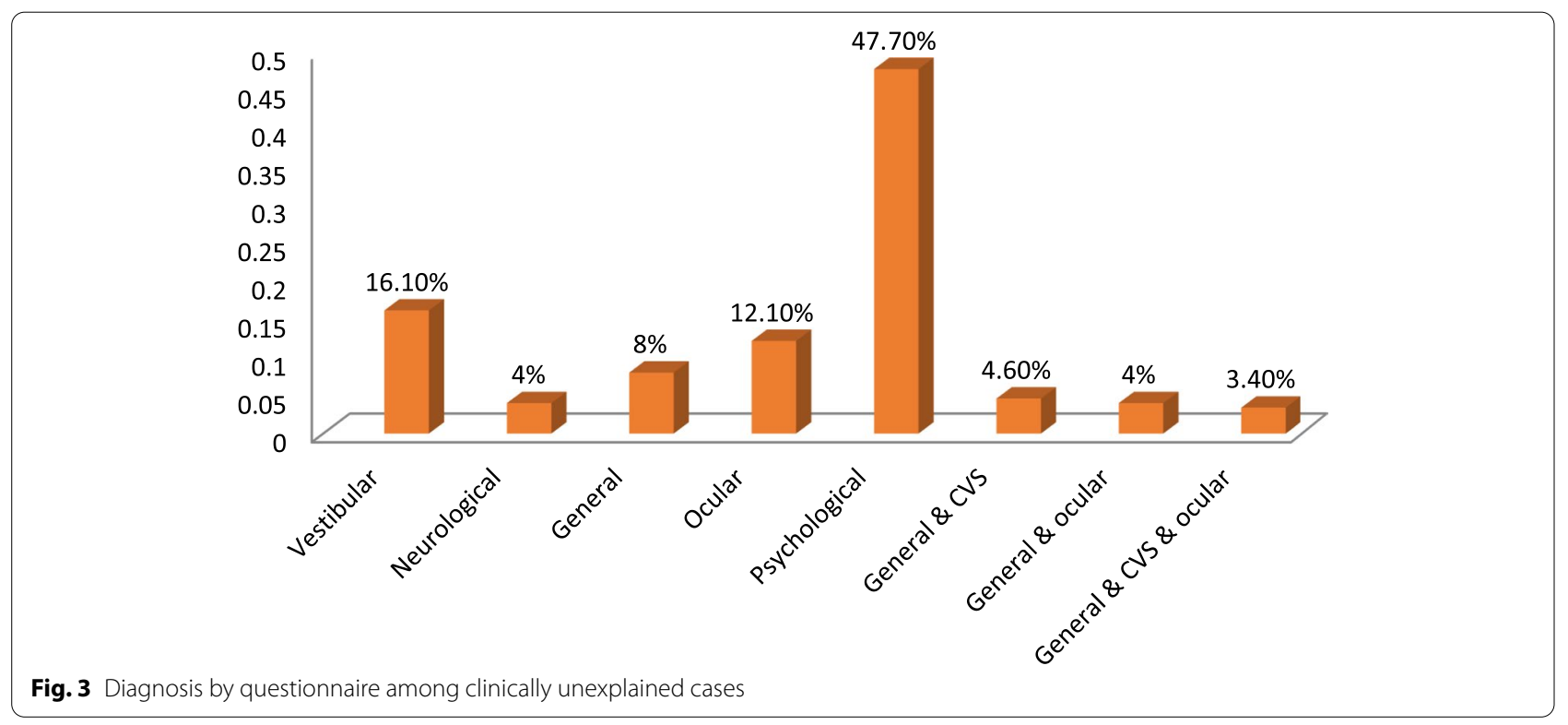

\section{Discussion}

Vertigo is not a rare condition in the paediatric age and, although there is wide variability in published prevalence data, it approaches nearly $10 \%$ in most clinical trials [4, 5]. It is estimated that $5-10 \%$ of children have at least one episode of vertigo before the age of 10 years, with $10 \%$ having at least one attack per year; in $51.5 \%$ of cases, the intensity of vertigo halts daily activities and in 25 to $50 \%$ of cases vertigo usually associated with any form of migraine $[2,4]$. In utmost clinical studies, differences in the prevalence of the single pathologies that may lead to vertigo can be perceived [10].
The study group consisted of 848 children, ranging from 6 to 18 years, with a mean age of $10.86 \pm 3.7$ years. Regarding sex distribution, $52 \%$ of the study group were females (441 cases) versus $48 \%$ males (407 cases). In the study conducted by Farid et al. [18], the number of children included in their study was only 40 cases. Their age ranged from 6 to 12 years with a mean age of $10.25 \pm 2.18$ years, and their sex distribution was $60 \%$ for females and $40 \%$ for males. On the other hand, the study of Riina et al. [2] involved 119 children, ranging from 7 months to 17 years, with a mean age of 10.9 years. Their sex distribution was $52.9 \%$ for females and $47.1 \%$ for males, and the 
Table 9 Comparisons of types of hearing loss in different dizziness causes

\begin{tabular}{|c|c|c|c|c|c|c|}
\hline \multirow[t]{2}{*}{ Variables } & \multicolumn{2}{|c|}{ Unexplained $(n=174)$} & \multicolumn{2}{|c|}{ Explained $(n=674)$} & \multirow[t]{2}{*}{$p$-value } & \multirow[t]{2}{*}{ Sig. } \\
\hline & No. & $\%$ & No. & $\%$ & & \\
\hline \multicolumn{7}{|c|}{ Right side hearing } \\
\hline Normal & 154 & $88.5 \%$ & 424 & $62.9 \%$ & $<0.05$ & $S$ \\
\hline Conductive & 0 & $0 \%$ & 162 & $24 \%$ & & \\
\hline Sensorineural & 20 & $11.5 \%$ & 82 & $12.2 \%$ & & \\
\hline Mixed & 0 & $0 \%$ & 6 & $0.9 \%$ & & \\
\hline \multicolumn{7}{|l|}{ Left side hearing } \\
\hline Normal & 154 & $88.5 \%$ & 423 & $62.8 \%$ & $<0.05$ & $S$ \\
\hline Conductive & 0 & $0 \%$ & 192 & $28.5 \%$ & & \\
\hline Sensorineural & 20 & $11.5 \%$ & 53 & $7.9 \%$ & & \\
\hline Mixed & 0 & $0 \%$ & 6 & $0.9 \%$ & & \\
\hline
\end{tabular}

Table 10 Comparison of Pediatric Dizziness Questionnaire scores in different dizziness causes

\begin{tabular}{|c|c|c|c|c|c|}
\hline \multirow[t]{2}{*}{ Variables } & \multicolumn{2}{|c|}{$\begin{array}{l}\text { Unexplained }(n= \\
\text { 174) }\end{array}$} & \multicolumn{2}{|c|}{$\begin{array}{l}\text { Explained }(n= \\
674)\end{array}$} & \multirow[t]{2}{*}{$p$-value } \\
\hline & Mean & SD & Mean & SD & \\
\hline Vestibular & 10.3 & 3.6 & 7.8 & 2.6 & $<0.01$ \\
\hline Neurological & 9.7 & 3.1 & 4.5 & 2.9 & $<0.01$ \\
\hline General & 11.1 & 2.9 & 6.3 & 4.9 & $<0.01$ \\
\hline CVS & 9.6 & 2.6 & 2.8 & 3.3 & $<0.01$ \\
\hline Ocular & 9.8 & 3.2 & 1.5 & 3.2 & $<0.01$ \\
\hline Psychological & 12.5 & 3.9 & 0.58 & 2.1 & $<0.01$ \\
\hline Total score & 62.9 & 10.3 & 23.5 & 8.7 & $<0.01$ \\
\hline
\end{tabular}

Table 11 Agreement in diagnosis by paediatric dizziness questionnaire and the final diagnosis reached by full assessment among cases with a possible diagnosis

\begin{tabular}{lll}
\hline Variables $(\boldsymbol{n}=\mathbf{6 7 4})$ & Agreement & \\
\cline { 2 - 3 } & Number & $\%$ \\
\hline Vestibular & 259 & $30.5 \%$ \\
Neurological \& vestibular & 102 & $12 \%$ \\
General & 56 & $6.6 \%$ \\
Vestibular \& general & 43 & $5.1 \%$ \\
Neurological & 42 & $4.9 \%$ \\
CVS & 14 & $1.7 \%$ \\
Vestibular \& cardiac & 12 & $1.4 \%$ \\
Total agreement & 528 & $78.3 \%$ \\
Total disagreement & 146 & $21.7 \%$ \\
\hline
\end{tabular}

study conducted by Lee et al. [20], involved 411 children, with their age ranging from 9 months to 18 years, with a mean age of 12.9 years, and their sex distribution was
$56 \%$ for females and $54 \%$ for males. These significant differences in demographic features and the number of children included in each study may be the reason for the variations in the results.

At the beginning of the study, a full detailed structured history was taken from all the patients and their guardians, filling in the paediatric dizziness questionnaire. Structured history taking is the most important diagnostic tool. It provides information about the type and severity of the complaints and the overall disability to prevent the consequences of balance disorders by implementing early therapeutic options [21].

Regarding hearing assessment, $61.7 \%$ of cases (523 cases) had bilateral within normal peripheral hearing threshold levels, $24.9 \%$ had conductive hearing loss, either unilateral $(8 \%)$ or bilateral $(16.9 \%)$, followed by $12.7 \%$ for sensorineural hearing loss either unilateral $(4.8 \%)$ or bilateral $(7.9 \%)$ and $0.7 \%$ for mixed hearing loss. There was a statistically significant difference with a $p$-value $<0.05$ between explained and unexplained groups as regards hearing loss with a higher percentage of normal PTA on both sides among patients with unexplained dizziness and a higher percentage of conductive hearing loss on both sides among patients with possible diagnosis, most of them were due to otitis media. Riina et al. [2], reported that most of their patients (77\%) had normal audiograms. On the other hand, Farid et al. [18], stated that only $50 \%$ of their patients had normal audiograms.

Moreover, there is a statistically significant difference with a $p$-value $<0.05$ between explained and unexplained groups as regards hearing loss with a higher percentage of normal PTA on both sides among patients with unexplained dizziness and a higher percentage of conductive hearing loss on both sides among patients with possible diagnosis, most of them were due to otitis media. 


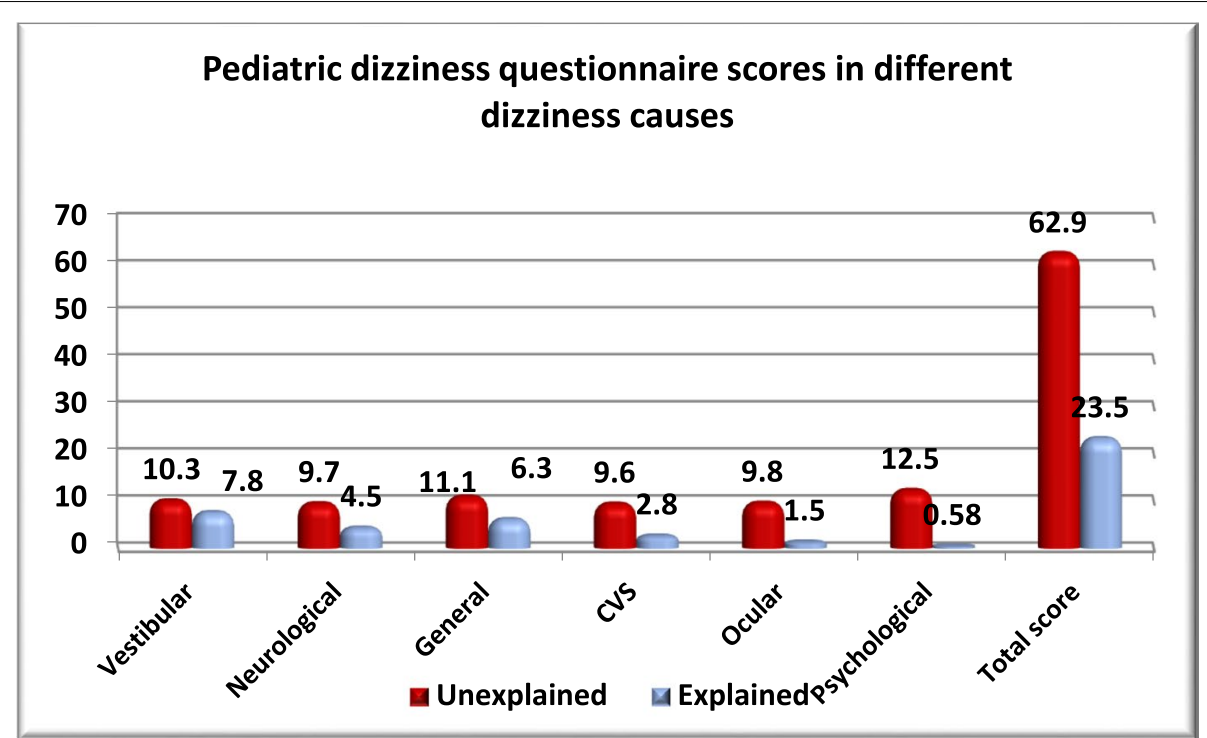

Fig. 4 Agreement in diagnosis by paediatric dizziness questionnaire and the final diagnosis reached by full assessment among cases with a possible diagnosis

In the current study, otitis media-related dizziness was reported by 203 children (23.9\%), either alone (11.1\%94 cases) or combined with other diseases such as DM, thalassemia, sickle cell anaemia, cardiovascular and respiratory disorders (12.85\%- 109 cases). However, most were recurrent middle ear infections or chronic middle ear effusion. Riina et al. also found that (OME) is one of the most common causes of balance disturbance in children. They also reported that children with OME are more visually dependent on balance than healthy children. Bhandari and Gowasami [22] reported that otitis media and middle ear effusions are the most common causes of vestibular disturbances in children. Otitis media shows apparent symptoms of infection, which makes diagnosis very easy. So, most researchers exclude otitis media from the differential diagnosis of vertigo in children. Peripheral vestibular abnormalities were noted in $34 \%$ of children with otitis media with effusions in our study group (69 cases), especially in post head shaking and positional tests. Opposing our results, Said et al. [23], reported peripheral vestibular abnormalities in 64\% (32 cases) of children with bilateral OME. On the other hand, Engel-Yeger et al. [24], found no significant abnormalities in VNG testing. This disparity in the results of VNG in children may reflect the lack of normative data for children. Moreover, children do not tolerate sitting still with their heads stabilized long enough for the examination to be carried out.

Accordingly, the audiological evaluation must be considered in all dizzy children since otitis media with effusion is a common condition that causes an imbalance in children.
In our current study, vertigo diagnosis was based on standard published criteria, detailed history, laboratory tests, imaging studies, and consultation documents from other specialties: neurology, ophthalmology, cardiology, haematology, and endocrinology. The most common causes of dizziness in children encountered were otitis media that represented (23.9\%-- 203 cases) either alone $(11.1 \%-94$ cases) or combined with another disease $(12.85 \%-109$ cases), followed by benign paroxysmal vertigo of childhood (17.6\% - 149 cases) and vestibular migraine (11.2\% - 95 cases). Similar findings were obtained in the study conducted by Riina et al., two on 119 children with a primary complaint of vertigo. Benign paroxysmal vertigo of childhood (BPVC) accounted for 19.3\% of cases, followed by vestibular migraine $14.3 \%$ of cases and otitis media-related dizziness $10.1 \%$ of cases. Choung et al. [25], identified BPVC as the second most common cause of vertigo in children (25.5\%) after migraine (30.9\%). Ravid et al. [26] had similar findings (16\% vs. 39\%). Balatsouras et al., five recorded BPVC in $16.7 \%$, and migraine in $20.4 \%$. Niemensivu et al., three and Ozeki et al. [27], suggested that benign paroxysmal vertigo is closely linked to migraine and could be interpreted as a migraine precursor. According to Lee et al. [20], BPVC and vestibular migraine are the leading causes of dizziness among children and adolescents, and the incidence of disease by age shows uneven distributions. BPVC was the most common in the preschool age group, followed by vestibular migraine, whereas, in the school-age group, BPVC and vestibular migraine were equally common. Vestibular migraine was the most common in adolescents. 
In the current study, $52.4 \%$ of cases of vestibular migraine and benign paroxysmal vertigo of childhood had a positive family history of migraine in first-degree relatives (128 cases out of 244 cases). Lateef et al. [28], also reported that a family history of migraine is the most potent and reliable risk factor for migraine, with a two- to three-fold more significant risk of migraine among relatives of migraine patients than controls. Family history of disorders should be considered for clinical assessment and treatment decisions and identifying migraine underlying genetic factors.

Although McCaslin et al. [8] found that migraine involvement of the brainstem and vestibular pathways may be responsible for the incidence of periodic vestibular symptoms in a wide range, it is not precisely known the pathogenesis of vestibulopathy and its association with migraine. Dizziness could be caused by symmetrical brainstem involvement, whereas an asymmetrical one could cause true vertigo.

Abnormal vestibular findings detected in our migraine patients in this study were positional nystagmus, posthead-shaking nystagmus, unilateral peripheral vestibular hypofunction as well as ocular motor abnormalities. The results revealed peripheral and central vestibular involvement, but it was challenging to draw a sharp distinction between the two possibilities. Similarly, Espinosa et al. [29] also found that migrainous vertigo may consist of both peripheral and central impairment. Central spontaneous nystagmus and ocular-motor abnormalities could only be explained by internal auditory artery ischemia, but it may manifest infarction in the anterior inferior cerebellar artery territory. Therefore, it is speculated that migraine and stroke are not just comorbid conditions. Nevertheless, instead, migraine is possibly an independent cerebrovascular risk factor, particularly among young people [30].

In terms of vestibular testing, 159 children refused to continue. They primarily declined to complete caloric testing and oculomotor evaluation, highlighting the importance of exploring alternatives to VNG. Caloric testing gave $22.4 \%$ abnormal results, but it proved to be one of the most challenging tests in our study group. Most children moved a lot during caloric tests, making it difficult for the physician to complete it correctly. Salami et al. [31], reported these apparent difficulties in performing a standard adult procedure of VNG on children. On the other hand, positional \& positioning tests did not give a high percentage of abnormalities (positional test $9.8 \%$, positioning test $5.5 \%$ ), but they contribute either to exclude or reach a diagnosis.

In the present study, we found only $4.4 \%$ of cases diagnosed as vestibular neuritis or labyrinthitis suspected from the history and canal paresis on VNG testing. Our finding agrees with Ralli et al., [32],who stated that vestibular neuritis rarely affects children below ten years. Choung et al. [25], reported that vestibular neuritis caused vertigo in only $2 \%$ of 52 paediatric patients. Ravid et al. [26], reported $14 \%$ of their 62 paediatric patients. The difference in results may be attributed to the variability of the number of cases.

We also found only 5 cases suspected with perilymph fistula as concluded from history and + ve fistula test done while all vestibular function tests were normal. This finding agreed with Erberk et al. [33], who found that perilymphatic fistula (PLF) is rarely seen in children, probably because of underestimation of this finding.

Only 3 cases $(0.35 \%)$ were suspected of Meniere's disease or labyrinthine hydrops in the present study. Similar results were obtained by Ravid et al., [26], who found no cases of Meniere's in their 62 patients. Also, Balatsouras et al. [5], reported only 1 case out of 50 patients, and Erbek et al. [33], found again only 1 of their 50 paediatric patients with Meniere's disease. Collectively, VNG findings agreed with Szirmai [34], Uneri \&Turkdogan [35], who stated that VNG together with history and PTA were necessary to reach a correct diagnosis.

Epilepsy represented 2.2\% of cases in the current study. Similar results were obtained by Riina et al. [2], who reported only 3 cases out of 119 children. Also, Choung et al. said that seizures caused vertigo in only $2 \%$ of their 55 paediatric patients, Erbek et al. [33] found 6\% of their paediatric patients with epilepsy, and Ravid et al., recorded only $3 \%$ of their patients with epilepsy. Only 8 cases $(0.94 \%)$ were diagnosed with brain tumours, which was agreed with Choung et al. [25],, who reported that a CP angle tumour caused vertigo in only $2 \%$ of their 52 paediatric patients. In the same way. Brain tumours are sporadic causes of vertigo in children, with a prevalence of just around 5/100,000. Approximately $1 \%$ of all paediatric intracranial tumours are in the posterior fossa, which may cause dizziness or imbalance [16].

Cardiovascular disorders were detected in $3.1 \%$ of cases (26 cases) either alone (14 cases) or combined with other diseases (12 cases). Both occult cardiac arrhythmias and acute myocardial infarctions could manifest as dizziness. Changes in blood pressure and changes in pulse are common in adolescents. Ravid et al. [26], reported orthostatic hypotension in $9 \%$ of their 62 paediatric dizzy patients and syncope in $3 \%$ of his cases.

We found that $1.8 \%$ (15 cases) of our study group suffered from ocular problems, in agreement with Erberk et al. [33], who found that various ophthalmological disorders could appear in children due to the ongoing development of the visual system during the first decade of life. We depend mainly on history, consultation documents from an ophthalmologist, and exclusion of other causes 
to reach our diagnosis. Children with vertigo and dizziness but with normal neurological findings and no apparent vestibular abnormalities after vestibular tests should undergo complete ocular testing to identify any ocular abnormality as a potential cause of vertigo and dizziness.

Other medical aetiologies that cause dizziness are diverse but primarily include hypertension, infection, hypoglycemia, and medications. Among our cases, 13.9\% (118 cases) suffered from widespread systemic diseases like anaemia, DM, and other endocrinal disorders either alone (56 cases) or combined with other conditions (62 cases). The diagnosis was confirmed from their medical history reports and laboratory tests.

In our study group, $10.5 \%$ of cases (89 cases) were categorized as psychogenic dizziness by paediatric dizziness questionnaire. Similarly, Erbek et al. [33] reported that $10 \%$ of paediatric dizzy patients had 'psychogenic vertigo'. Also, six of 119 were diagnosed with psychogenic dizziness in the study of Riina et al. [2]. Depression, conversion, and somatization were identified as the most frequent causes. Historical findings indicate psychogenic disorders are the inability to attend school despite normal physical examination and observing unusual interactions with guardians (Emeriglu et al., 2004).

In the present study, we could not reach a possible diagnosis in $20.5 \%$ of cases (174 cases). Similarly, Choung et al. [25] reported that $18.2 \%$ of their paediatric dizzy patients were 'unclassified'. Such patients typically need to be followed more closely than patients with a precise diagnosis. Empirical medication trials, psychiatric consultation, and vestibular physical therapy could be valuable options.

In the current study, the diagnosis by paediatric dizziness questionnaire was agreed with the final diagnosis reached by full assessment in about $78.3 \%$ of cases with a possible diagnosis. Ravid et al. [26], also reported that the sensitivity of the questionnaire to get the correct diagnosis was $92 \%$. Shabana et al. [11], also stated that in $75 \%$ of cases, the diagnostic categories of the questionnaire matched the diagnosis on referral. Its sensitivity in vestibular diagnosis was $88.9 \%$. The questionnaire seems to be an appropriate tool for diagnosing dizzy children, with a scoring system that can categorize dizzy children by the systems affected. The proposed standardized questionnaire could be a good and simple diagnostic tool for children with vertigo or dizziness.

Vertigo in children could be seen due to many causes. These include trauma, infections, migraine, central nervous system disorders (usually cerebellar and brainstem), benign paroxysmal positional vertigo (BPPV), vestibular neuritis due to viral infections, and psychosomatic disorders [21].
Vertigo in children must be given adequate consideration since it can be the only symptom of a broad spectrum of diseases, including central nervous system neoplasms or inner ear pathologies. Moreover, especially in childhood, vertigo and dizziness can produce numerous prescriptions for useless and expensive testing without obtaining any help in therapeutic decisionmaking [10].

Children are challenging to evaluate. Not only do 'vertigo in this critical age' has a different differential diagnosis, but the affected children respond relatively well to the treatment and recover quicker than the adults. Thus, it is most important to determine whether a vestibular disorder exists in a child to prescribe the appropriate rehabilitative therapy, which can improve those children's general well-being and quality of life.

\section{Conclusion}

Diagnosing dizziness in children is challenging; nevertheless, a structured history taken in conjunction with a vestibular examination is crucial for establishing an accurate diagnosis. The questionnaire appears to be an evenhanded tool for diagnosing dizzy children.

\section{Recommendations}

The proposed structured questionnaire should be incorporated in the diagnostic process of dizzy children and is likely to eliminate the need for unnecessary costly evaluation. Besides a thorough and detailed history taking, a basic audiological evaluation, a bedside examination, VNG, Video Head Impulse test, and VEMP for children to avoid dropouts found in the current study.

\section{Supplementary Information}

The online version contains supplementary material available at https://doi. org/10.1186/s43163-022-00222-0.

\section{Additional file 1}

\section{Acknowledgements}

I wish to show my appreciation to Dr. Asmaa Younes, who offered valuable help in the statistics we used in our work, and all our colleagues in the ENT Department, Faculty of Medicine, Fayoum University.

\section{Authors' contributions}

All authors contributed to the design and implementation, of the research, to the analysis of the results, and to the manuscript's writing. Dr. MA, Dr. RS and Dr. MM were responsible for the audiological and vestibular assessment. Prof SG and Prof HA were responsible for analyzing the results and writing the discussion. Also, the manuscript has been read and approved for submission by all authors and has not been submitted or published elsewhere.

Funding

None.

Availability of data and materials

All datasets used are available. 


\section{Declarations}

Ethics approval and consent to participate

The study was approved by the local research ethics board of Fayoum University on May 15th, 2016 (reference number D 86); written informed consent was obtained from all patients' guardians to participate in this work.

\section{Consent for publication}

All patients' guardians obtained

written informed consent to publish this work.

\section{Competing interests}

The authors declare they have no competing interests, no financial or nonfinancial conflict of interest.

\section{Author details}

${ }^{1}$ Department Faculty of Medicine, Helwan University, Cairo, Egypt. ${ }^{2}$ ENT Department, Faculty of Medicine, Fayoum University, Fayoum 63514, Egypt.

Received: 3 November 2021 Accepted: 21 January 2022

Published online: 14 February 2022

\section{References}

1. Luxon L, Pagarkar W (2008) The dizzy child Chapter 46. In: Graham JM, Scadding GK, Bull PD (eds) Pediatric ENT, 1st edn. Springer-Verlag, Germany, pp 460-473

2. Riina $N$, Ilmari $P$, Kentala $E$ (2005) Vertigo and imbalance in children a retrospective study in a Helsinki University otorhinolaryngology clinic. Arch Otolaryngol Head Neck Surg 131:996-1000

3. Niemensivu R, Kentala E, Wiener-Vacher S et al (2007) Evaluation of vertiginous children. Eur Arch Otorhinolaryngol 264:1129-1135

4. Wiener-Vacher SR (2008) Vestibular disorders in children. Int J Audiol 47:578-583

5. Balatsouras DG, Kaberos A, Assimakopoulos D et al (2007) Etiology of vertigo in children. Int J Pediatr Otorhinolaryngol 71:487-494

6. Erbek SH, Erbek SS, Yilmaz I et al (2006) Vertigo in childhood: a clinical experience. Int J Pediatr Otorhinolaryngo 70:1547-1554

7. Jahn K, Langhagen T, Schroeder AS et al (2011) Vertigo and dizziness in childhood update on diagnosis and treatment. Neuropediatrics 42:129-134

8. McCaslin DL, Jacobson GP, Gruenwald JM (2011) The predominant forms of vertigo in children and their associated findings on balance function testing. Otolaryngol Clin North Am 44:291-307

9. Casselbrant ML, Mandel EM (2005) Balance disorders in children. Neurol Clin 23(3):807

10. Casani AP, Dallan I, Navari E et al (2015) (2015): Vertigo in childhood: proposal for a diagnostic algorithm based upon clinical experience. Acta Otorhinolaryngol Ital. 35(3):180-185

11. Shabana MI, Dabbous AO, Hosni NA et al (2012) Can scoring of symptoms in dizzy children aid the categorization of causes of dizziness for accurate referral? Egypt J Otolaryngol 28:214-233. https://doi.org/10.7123/01.EJO. 0000418071.01257.ba

12. Soliman S (1976) Speech discrimination audiometry using Arabic phonetically balanced words. Ain Shams Med J 27:27-30

13. Soliman S, Fathalla A, Shehata M (1985) Development of Arabic staggered spondee words (SSW) test. Proceedings of the 8th Ain Shams Medical Congress Egypt, vol 2, pp 1220-1246

14. Kuo C, Pang L, Chang R (2008) Vertigo- Assessment in general practice. Aust Fam Phys 37(5):341-347

15. Eviatar L (1994) Dizziness in children. Otolaryngol Clin North Am. 27:557-571

16. Hain TC, Cherchi M (2019) Migraine associated vertigo. Adv Otorhinolaryngol 82:119-126

17. Prakash S (2018) The international classification of headache disorders: Anything new in 3rd edition? J Integr Health Sci 6:1-2

18. Farid A, Shabana M, El-Kosht M et al (2012) Difficulties encountered during vestibular testing of pediatric patients (aged 6-12 years). Med J Cairo Univ 80(2)
19. Strupp M, Hüfner K, Sandmann R et al (2011) Central oculomotor disturbances and nystagmus: a window into the brainstem and cerebellum. Dtsch Arztebl Int. 108(12):197-204

20. Lee JD, Kim CH, Hong SM et al (2017) Prevalence of vestibular and balance disorders in children and adolescents according to age: a multicenter study. Int J Pediatr Otorhinolaryngol 94:36-39

21. Walther LE (2017) Current diagnostic procedures for diagnosing vertigo and dizziness. GMS Curr Top Otorhinolaryngol Head Neck Surg 16:Doc02

22. Bhandari A, Goswami A (2019) Differential diagnosis of vertigo in children. Ann Otol Neurotol 2(01):21-26

23. Said EA, Ahmed MK, Mohamed ES (2015) Role of vestibular testing in deciding treatment strategies for children with otitis media with effusion. Egyptian J Ear Nose Throat Allied Sci 16(2):151-159

24. Engel-Yeger B, Golz A, Parush S (2004) Impact of middle ear effusion on balance performance in children. Disabil Rehabil 26(2):97-102

25. Choung YH, Park K, Moon SK et al (2003) Various causes and clinical characteristics in vertigo in children with normal eardrums. Int J Pediatr Otorhinolaryngol. Aug 67(8):889-894

26. Ravid S, Bienkovski R, Eviatar L (2003) A simplified diagnostic approach to dizziness in children. J Pediatr Neurol 29:317-320 Res 67:13-17

27. Ozeki H, Iwasaki S, Murofushi T (2008) Clinical features of vertigo and dizziness in childhood and adolescence with a special reference to benign recurrent vertigo. Equilibrium Res 67:13-17

28. Lateef TM, Cui L, Nakamura E et al (2015) Accuracy of family history reports of migraine in a community-based family study of migraine. Headache J Head Face Pain 55(3):407-412

29. Espinosa-Sanchez JM, Lopez-Escamez JA (2015) New insights into the pathophysiology of vestibular migraine. Front Neurol 6:12

30. Guidetti D, Rota E, Morelli N, Immovilli P (2014) Migraine and stroke: "vascular" comorbidity. Front Neurol 8(5):193. https://doi.org/10.3389/fneur. 00193

31. Salami A, Dellepiane M, Mora R et al (2005) Electronystagmography finding in children with peripheral and central vestibular disorders. Int J Pediatr Otorhinolaryngol 70(1):13-18

32. Ralli G, Atturo F, Filippis C (2009) Idiopathic benign paroxysmal vertigo in children, a migraine precursor. Int J Pediatr Otorhinolaryngol 73:16-18

33. Erbek S, Erbek S, Ismail Yilmaz I et al (2006) Vertigo in childhood: a clinical experience. Int J Pediatr Otorhinolaryngol 70:1547-1554

34. Szirmai A (2010) Vestibular disorders in childhood and adolescents. Eur Arch Otorhinolaryngol 267(11):1801-1804

35. Uneri A, Turkdogan D (2003) Evaluation of vestibular functions in children with vertigo attacks. Arch Disease Child 88:510-511

\section{Publisher's Note}

Springer Nature remains neutral with regard to jurisdictional claims in published maps and institutional affiliations.

\section{Submit your manuscript to a SpringerOpen ${ }^{\circ}$ journal and benefit from:}

- Convenient online submission

- Rigorous peer review

- Open access: articles freely available online

- High visibility within the field

Retaining the copyright to your article

Submit your next manuscript at springeropen.com 